\title{
PHOTON AND NEUTRINO-EMISSION FROM SHOCKWAVES IN ACTIVE GALACTIC NUCLEI
}

\author{
Peter L. Biermann \\ Max-Planck-Institut für Radioastronomie \\ Auf dem Hügel 69 \\ 5300 Bonn 1, F.R.G.
}

\begin{abstract}
Many active galactic nuclei and/or associated jets and hot spots show a sharp cutoff in their nonthermal emission near $\sim 310^{14} \mathrm{~Hz}$. Using the first order Fermi theory for particle acceleration at shocks we demonstrate that synchrotron emission and shock acceleration combine to give a maximum cutoff near to the observed frequency. We explore consequences: First, CCD-observations of the M87 jet demonstrate that the cutoff frequency decreases along the jet. Second, the detailed shape of the cutoff spectrum depends on the precise model for the turbulent wave field. Thirdly, an $\mathrm{X}$-ray synchrotron contribution from $\mathrm{p}-\mathrm{p}$ and $\mathrm{p}-\gamma$ interactions exists which can match observed AGN spectra. Finally we apply the model to interpret the origin of very high energy cosmic rays, to the spectral behaviour of quasars and BL Lac's and deduce neutrino emission spectra of active galactic nuclei.
\end{abstract}

\section{THE CUTOFF NEAR $310^{14} \mathrm{~Hz}$}

Numerous active galactic nuclei are observed to have both compact and extended jets. These jets often show a knotty structure, terminating in some cases in a hot spot inside a radio lobe. Optical and infrared observations of active nuclei have demonstrated that many BL Lac's, OVVs and red quasars have a sharp cutoff in their continuum radiation near 3 $10^{14} \mathrm{~Hz}$. A similar cutoff has been found in the emission from knots in jets as well as hot spots.

Here we consider first-order Fermi acceleration at a shock. Energetic particles can be scattered by magnetic irregularities on both sides of a shock which forms a perpetually converging flow. Putting $\tau_{\text {acc }}=\tau_{\text {syn }}$ leads then to maximum Lorentz-factors of protons and electrons of $\gamma_{p \text {, max }}=6.0$ $10^{10}(\mathrm{~b} / \mathrm{B})^{1 / 2} \quad \mathrm{U}_{1} / \mathrm{c}$ and $\gamma_{\mathrm{e}, \max }=1.810^{4}(\mathrm{~b} / \mathrm{B})^{1 / 2} \quad \mathrm{U}_{1} / \mathrm{c}$ for $\mathrm{B}=5 / 3$ (Kolmogorov). Here $b$ is the fractional magnetic energy in waves, and $B$ the magnetic field strength. Thus, proton acceleration in shocks may explain the origin of high energy cosmic rays. Adding photon interaction losses for protons, gives an upper limit to the synchrotron emission frequency of $v^{*}=310^{14}\left[3 b\left(U_{1} / c\right)^{2}\right] \mathrm{f}(\mathrm{a}) \mathrm{Hz}$ with $\mathrm{P}(\mathrm{a})=(1+\mathrm{Aa})^{1 / 2} /(1+\mathrm{a})^{3 / 2}$. Here $\mathrm{a}$ is the photon energy density relative to $B^{2} / 8 \pi$, and $A \simeq 200$. Thus a real maximum to the synchrotron emission frequency exists and is at $v_{\max }^{*}=$ $1.910^{15}\left[3 \mathrm{~b}\left(U_{1} / \mathrm{c}\right)^{2}\right] \mathrm{Hz}$. Observational checks as regards $\mathrm{p}-\mathrm{p}$ interactions, lengthscales and timescales can be made. 


\section{HIGH ENERGY PHOTONS AND NEUTRINOS}

The neutrino-luminosity $\mathrm{L}_{v}$ can be calculated by integrating the emissivity per volume along a streamline downstream from a shock, and then comparing with the synchrotron luminosity calculated in the same manner. As already noted by Biermann and Strittmatter (1987) this ratio depends critically on the ratio of the energy density in protons to that in electrons $a_{p} / a_{e}$. For reasonable parameter values, the ratio of all involved quantities is very close to unity, and so $L_{v} / L_{s y n} \simeq a_{p} / a_{e}$. For nearrelativistic shocks the ratio $a_{p} / a_{e}$ might well be expected to be close to unity (Bell 1978) and so rather extreme neutrino-luminosities are expected for extreme active nuclei $(a \simeq 1)$. Eichler (1979) came to a similar conclusion using quite different physical arguments. The neutrino spectrum is $E_{2}{ }^{-1}$ for a protron $\gamma_{p}^{-2}$ and photon $\varepsilon^{-2}$ spectrum, and $E_{\mathcal{L}}^{-2}$ for a proton $\gamma_{p}^{-2}$ and a sharply peaked photon spectrum. Should the maximum distance L allowed along a stream-line lead to lower neutrino energies than the energy considered by the observer, then we have to multiply with a factor of $\sim E_{V}{ }^{-1}$ which leads to a number spectrum of $E_{V}{ }^{-2}$ or $E_{V}{ }^{-3}$, respectively. We adopt $\mathrm{E}_{V}{ }^{-2}$ as the neutrino-spectrum for reference. Consider then the quasar $3 \mathrm{C} 273$ with a mm-Xray luminosity of $\sim 10^{48}$ erg $\mathrm{sec}^{-1}$. Putting the neutrino luminosity equal to this - mostly apparently nonthermal luminosity yields a neutrino spectrum $4 \quad 10^{-10} \quad \mathrm{E}_{\nu}^{-2} \mathrm{~cm}^{-2} \mathrm{sec}^{-1}$ with $\mathrm{E}_{\nu}$ measured in erg $(\sim \mathrm{TeV})$.

\section{FURTHER WORK}

Pérez-Fournon et al. (1988) obtained CCD-photometry of the M87 Jet in two bands and so derived the spatial variation of the optical spectral index. Such a variation can be translated into a change in cutoff frequency: The cutoff frequency decreases noticeably outwards, especially beyond the strong knot A. Klemens (1987) has shown, p-p and $p-y$ collisions may be important in the $X$-ray regime. Combining primary electron emission with secondary pair emission for the synchrotron radiation may explain the far infrared, optical, $X$-ray overall spectrum of nonthermal sources. Fritz (1988) made an important step towards a physical understanding of the cutoff spectrum by calculating exactly the distribution function of energetic electrons. Fritz demonstrated that for a scattering mean free path which rises with energy, the cutoff becomes sharper. Kirk, Schneider, Webb and Krülls have started to address relativistic shocks. Further observations are being made by Röser, Meisenheimer, Pérez-Fournon, Nieto and Fraix-Burnet.

ACKNOWLEGEMENT. I thank Drs. K.-D. Fritz, Y. Klemens, K. Meisenheimer, H. Meyer, I. Pérez-Fournon, Th. Schmutzler, P. Strittmatter, H.-J. Völk and Mssrs. D. Fraix-Burnet, W. Krülls and K. Mannheim for discussion.

\section{REFERENCES}

Bell, A.R.: 1978, M.N.R.A.S. 182, 147

Biermann, P.L., Strittmatter, P.A.: 1987 Astrophys. J. 322, 643

Eichler, D.: 1979, Astrophys. J. 232, 106

Fritz, K.D.: 1988, Astron. Astrophys. (submitted)

Klemens, Y.: 1987, Proceed. Regional IAU Meeting, Prague, p. 391

Pérez-Fournon, I. et al.: 1988 Astrophys. J. 329, L81 